\title{
On a queer binomial sum
}

\author{
R. Denk, R. Warlimont
}

Abstract. In this paper the binomial sum

$$
S_{n}(r)=\sum_{m=0}^{n}\left(\begin{array}{c}
n \\
m
\end{array}\right) \frac{(-1)^{m}}{m^{r}+1} \quad(r>0, n \in \mathbb{N})
$$

is investigated. It turns out that the behaviour of this sum for $n \rightarrow \infty$ depends on the parameter $r$ and changes dramatically at the values $r=1$ and $r=2$. In particular, for $r \geq 2$ we obtain an oscillatory behaviour while for $r<2$ the sequence $S_{n}(r)$ is monotonous at least for large values of $n$.

Mathematics Subject Classification (MSC 1991). 11B65

1. Introduction. For $n \in \mathbb{N}$ and real $r>0$ put

$$
S_{n}(r):=\sum_{m=0}^{n}\left(\begin{array}{l}
n \\
m
\end{array}\right) \frac{(-1)^{m}}{m^{r}+1}
$$

It is easy to show that $S_{n}(1)=\frac{1}{n+1}$ (see e.g. [1], Formula 0.155). In [2] the fact that $0<S_{n}(r) \leq \frac{1}{2}$ for $0<r<1$ was needed. The second author of the present paper furnished the proof: a formula for $S_{n}(r)$ has been established from which not only $S_{n}(r)>0$ can be read off but $S_{n}(r) \downarrow 0$ for $n \rightarrow \infty$ as well.

We resume this subject here and determine the behaviour of $S_{n}(r)$ for $n \rightarrow \infty$ for every fixed $r>0$. It is queer indeed. At the values $r=1$ and $r=2$ it takes unexpectedly a dramatic turn.

To formulate our results we have to introduce the following notation. For $r>0, r \notin \mathbb{N}$, and complex $z$ not belonging to the negative real axis we define $z^{r}:=\exp (r \log z)$ where $\log$ denotes the principal branch. The function $z^{r}+1$ has no zeros for $0<r<1$. For $r \geq 1$ it has the simple zeros $w_{k}(r)=\exp \left(\frac{i(2 k+1) \pi}{r}\right) \quad\left(-\frac{r+1}{2}<k \leq \frac{r-1}{2}\right)$. They occur in conjugate pairs. We single out $w(r):=w_{0}(r)=e^{i \pi / r}$ with maximal real part $\cos \frac{\pi}{r}$.

The main results are contained in the following theorem.

Theorem 1. Set $A_{n}(r)=0$ for $0<r<1$ and

$$
A_{n}(r):=\frac{1}{r} \sum_{k} \frac{n !}{\prod_{l=1}^{n}\left(l-w_{k}(r)\right)} \quad \text { for } r \geq 1 .
$$


For $r \in \mathbb{N}$ set $B_{n}(r)=0$. For $r>0, r \notin \mathbb{N}$, put

$$
\begin{aligned}
B_{n}^{*}(r) & :=\int_{0}^{\infty} \frac{u^{r}}{\left|1+u^{r} e^{i r \pi}\right|^{2}} d u \int_{0}^{\infty} e^{-u t}\left(1-e^{-t}\right)^{n} d t \\
B_{n}(r) & :=\frac{\sin r \pi}{\pi} B_{n}^{*}(r) .
\end{aligned}
$$

a) For every $r>0$ and $n \in \mathbb{N}$ we have

$$
\text { (1) } \quad S_{n}(r)=A_{n}(r)+B_{n}(r)
$$

b) For every $r>0, r \notin \mathbb{N}$, the asymptotics

(2) $\quad B_{n}^{*}(r)=\Gamma(r)(\log n)^{-r}\left(1+O\left((\log n)^{-\min \{1, r\}}\right)\right)$

hold for $n \rightarrow \infty$ with an O-constant depending only on $r$.

If we combine the results of Theorem 1 with

(3) $\frac{n !}{\prod_{l=1}^{n}(l-w)}=\Gamma(1-w) n^{w}+O\left(n^{\operatorname{Re} w-1}\right) \quad$ for $w \in \mathbb{C} \backslash \mathbb{N}$

we are able to explain the behaviour of $S_{n}(r)$ for $n \rightarrow \infty, r>0$ fixed. (Cf. also Fig. 1 for the behaviour of $S_{n}(r)$ for $0<r \leq 4$ and $1 \leq n \leq 100$.) In the following, the symbol $\downarrow$ denotes the monotonous decrease of a sequence.

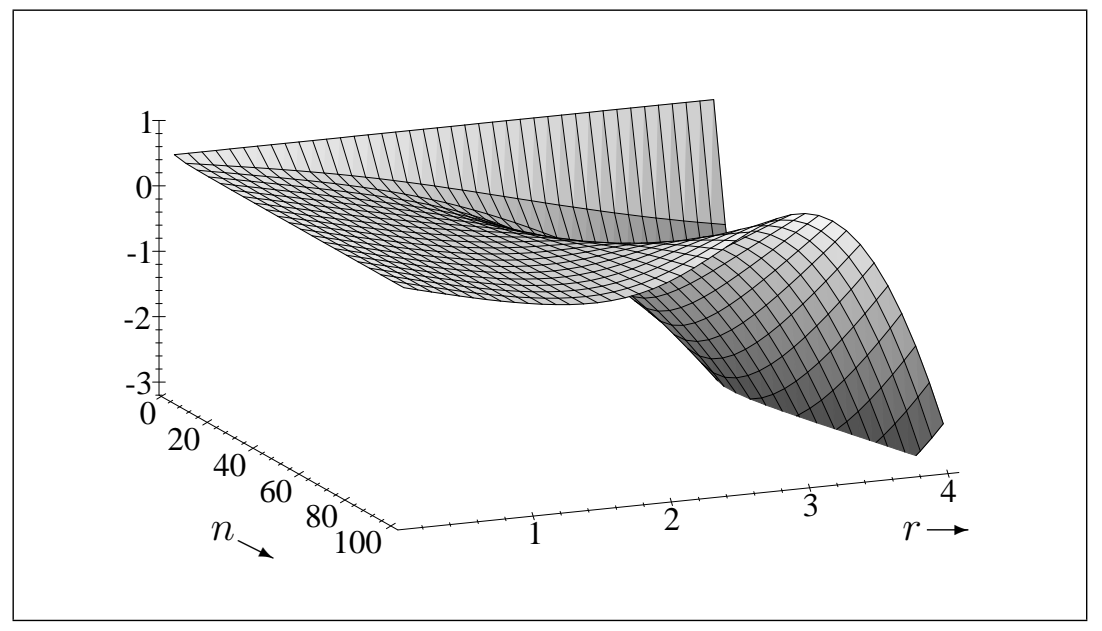

Figure 1: The function $S_{n}(r)$ for $0<r \leq 4$ and $1 \leq n \leq 100$. 
Theorem 2. The sum $S_{n}(r)$ has the following behaviour for fixed $r>0$ and $n \rightarrow \infty$ :

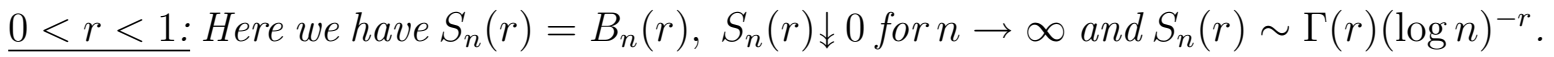
$1<r<2$ : In this case $S_{n}(r)=B_{n}(r)+O\left(n^{\cos \frac{\pi}{r}}\right),-S_{n}(r) \downarrow 0$ for $n \rightarrow \infty \quad\left(n \geq n_{0}(r)\right)$ and $-S_{n}(r) \sim \Gamma(r)(\log n)^{-r}$.

$r \geq 2:$ Put $a(r):=\sin \frac{\pi}{r}, b(r):=\arg \Gamma(1-w(r))$, and $c(r):=\frac{2}{r}|\Gamma(1-w(r))|$, where $\overline{w(r)}=e^{i \pi / r}$. Then

$$
s_{n}(r):=\frac{S_{n}(r)}{n^{\cos \frac{\pi}{r}}}=c(r) \cos [a(r) \log n+b(r)]+O\left(n^{-\delta(r)}\right)
$$

with some $\delta(r)>0$ which could be specified.

Now we use the fact that $\{\cos (a \log n+b): n \in \mathbb{N}\}$ is dense on $[-1,1]$ for $a \neq 0$ and $b \in \mathbb{R}$. To see this, we first remark that for every $a \neq 0$ one of the numbers $\frac{a}{2 \pi} \log 2$ or $\frac{a}{2 \pi} \log 3$ is irrational. Let $\frac{a}{2 \pi} \log q \notin \mathbb{Q}$ with $q=2$ or $q=3$. Then, by Kronecker's approximation theorem (see, for instance, [3], p. 150), for every $x \in \mathbb{R}$ and $\varepsilon>0$ there exist $m \in \mathbb{N}_{0}$ and $h \in \mathbb{Z}$ such that

$$
\left|\left(\frac{a}{2 \pi} \log q\right) m+\frac{b-x}{2 \pi}-h\right| \leq \frac{\varepsilon}{2 \pi}
$$

Let $y \in[-1,1]$ and $x \in \mathbb{R}$ with $\cos x=y$. Then for $n=q^{m}$ we obtain

$$
|\cos (a \log n+b)-y| \leq|(a \log n+b)-(x+2 \pi h)| \leq \varepsilon
$$

which shows the denseness result stated above. From this and from Theorem 2 we infer that for $r \geq 2$ the set $\left\{s_{n}(r): n \in \mathbb{N}\right\}$ is dense on $[-c(r), c(r)]$ and that

$$
\liminf _{n \rightarrow \infty} s_{n}(r)=-c(r), \quad \limsup _{n \rightarrow \infty} s_{n}(r)=c(r) .
$$

We mention that $\lim _{r \rightarrow \infty} c(r)=\frac{2}{\pi}$. In particular, for $r=2$ we get

$$
S_{n}(2)=\operatorname{Re} \frac{n !}{\prod_{l=1}^{n}(l-i)}=c(2) \cos [\log n+\arg \Gamma(1-i)]+O\left(\frac{1}{n}\right)
$$

$S_{n}(2)$ is dense on the interval $[-c(2), c(2)]$, and we obtain

$$
\begin{aligned}
& \liminf _{n \rightarrow \infty} S_{n}(2)=-c(2), \quad \limsup _{n \rightarrow \infty} S_{n}(2)=c(2), \\
& c(2)=\left(\frac{2 \pi}{e^{\pi}-e^{-\pi}}\right)^{1 / 2} .
\end{aligned}
$$




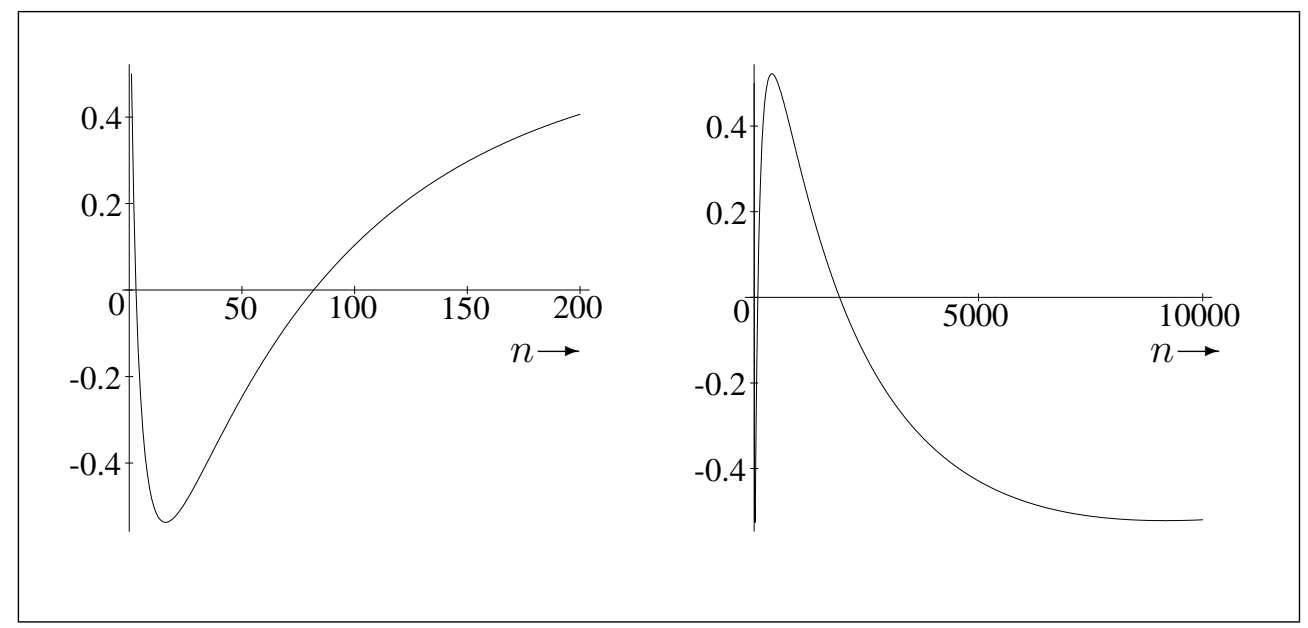

Figure 2: The function $S_{n}(2)$ for $1 \leq n \leq 200$ (left) and $1 \leq n \leq 10000$ (right).

In Fig. 2 one can see the oscillatory behaviour of $S_{n}(2)$ described mainly by the term $\cos [\log n]$ in $(4)$.

We proof (1) for $r \in \mathbb{N}$ in Section 2 and for $r>0, r \notin \mathbb{N}$, in Section 3. In Section 4 we prove (2).

The following identity will be used all the time: for $\operatorname{Re} \alpha<0, \operatorname{Re} \beta \leq 0$, and $m \in \mathbb{N}_{0}$ one has

(5) $\quad I(\alpha, \beta, m):=\int_{0}^{\infty} e^{\alpha t}\left(1-e^{\beta t}\right)^{m} d t=-\frac{m ! \beta^{m}}{\prod_{l=0}^{m}(\alpha+l \beta)}$.

This identity can be derived from [1], Formula 3.312, or proved directly by induction with respect to $m$.

2. The case $r \in \mathbb{N}$. We have

$$
\begin{aligned}
S_{n}(1) & =\sum_{m=0}^{n}\left(\begin{array}{c}
n \\
m
\end{array}\right)(-1)^{m} \int_{0}^{1} u^{m} d u=\int_{0}^{1}(1-u)^{n} d u=\frac{1}{n+1}, \\
S_{n}(2) & =\sum_{m=0}^{n}\left(\begin{array}{c}
n \\
m
\end{array}\right)(-1)^{m} \operatorname{Re} \int_{0}^{1} u^{i m} d u=\operatorname{Re} I(n) \text { with } \\
I(n) & :=\int_{0}^{1}\left(1-u^{i}\right)^{n} d u=\int_{0}^{\infty} e^{-v}\left(1-e^{-i v}\right)^{n} d v=I(-1,-i, n) \\
& =-\frac{n !(-i)^{n}}{\prod_{l=0}^{n}(-1-l i)}=\frac{n !}{\prod_{l=1}^{n}(l-i)},
\end{aligned}
$$

where we used (5). Now let $r \geq 3$. In this case we use the decomposition into partial 
fractions and get

$$
\frac{1}{z^{r}+1}=-\frac{1}{r} \sum_{k} \frac{w_{k}}{z-w_{k}}=-\frac{1}{r} \sum_{k} w_{k} \int_{0}^{\infty} \exp \left[\left(w_{k}-z\right) t\right] d t
$$

for $\operatorname{Re} z>\cos \frac{\pi}{r}$. In particular we obtain

$$
\frac{1}{m^{r}+1}=-\frac{1}{r} \sum_{k} w_{k} \int_{0}^{\infty} \exp \left[\left(w_{k}-m\right) t\right] d t \quad \text { for } m \in \mathbb{N} .
$$

From this we infer

$$
S_{n}(r)=1-\frac{1}{r} \sum_{k} w_{k} \int_{0}^{\infty} e^{w_{k} t}\left(\left(1-e^{-t}\right)^{n}-1\right) d t .
$$

Integrating by parts we find

(6) $\quad \int_{0}^{\infty} e^{w_{k} t}\left(\left(1-e^{-t}\right)^{n}-1\right) d t=\frac{1}{w_{k}}-\frac{n}{w_{k}} I\left(w_{k}-1,-1, n-1\right)$.

Therefore

$$
S_{n}(r)=\frac{1}{r} \sum_{k} n I\left(w_{k}-1,-1, n-1\right)=A_{n}(r) \quad \text { by }(5) .
$$

3. The case $r \notin \mathbb{N}$. Denote by $\mathbb{C}^{-}$the complex plane cut along the negative real axis. For $0<r<1$ the function $\frac{1}{z^{r}+1}$ is holomorphic on $\mathbb{C}^{-}$. For $r>1$ it is meromorphic there with simple poles at $w_{k}\left(-\frac{r+1}{2}<k \leq \frac{r-1}{2}\right)$.

Let $\operatorname{Re} z>1$. Then $|z|>1$. Therefore

$$
\frac{1}{z^{r}+1}=\sum_{j=1}^{\infty}(-1)^{j-1} z^{-r j}=\sum_{j=1}^{\infty} \frac{(-1)^{j-1}}{\Gamma(r j)} \int_{0}^{\infty} t^{r j-1} e^{-z t} d t
$$

from which we infer

$$
\frac{1}{z^{r}+1}=\int_{0}^{\infty} f(r, t) e^{-z t} d t \quad(\operatorname{Re} z>1)
$$

with $f(r, t):=\sum_{j=1}^{\infty} \frac{(-1)^{j-1}}{\Gamma(r j)} t^{r j-1} \quad(t>0)$.

By the inversion formula of Laplace $([4],(1.4 .2))$ we get

$$
f(r, t)=\lim _{T \rightarrow \infty} \frac{1}{2 \pi i} \int_{c-i T}^{c+i T} \frac{e^{t z}}{z^{r}+1} d z \quad(c>1, t>0) .
$$

Let $X \geq 2$ be given. We deform the interval $[c-i T, c+i T]$ into the following contour: 


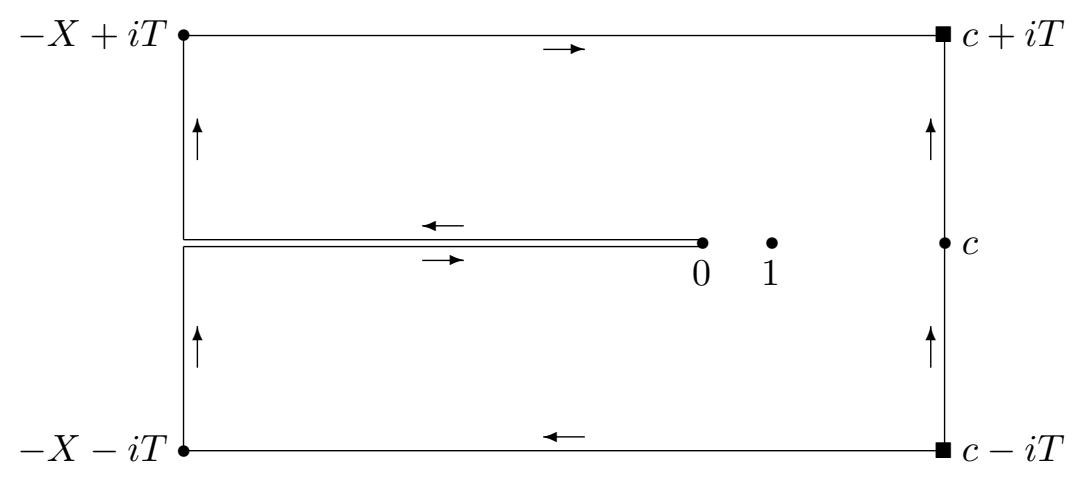

We apply the theorem of residues and take first the limit $X \rightarrow \infty$ and then the limit $T \rightarrow \infty$. This leads to the representation

(8) $f(r, t)=g(r, t)+h(r, t)$

where

$$
\begin{aligned}
g(r, t) & :=\sum_{k} \operatorname{Res}_{w_{k}} \frac{e^{t z}}{z^{r}+1} \quad(r>1), \\
h(r, t) & :=\frac{1}{2 \pi i} \int_{\stackrel{\leftrightarrows}{\rightarrow} 0} \frac{e^{t z}}{z^{r}+1} d z .
\end{aligned}
$$

We find

(9) $g(r, t)=-\frac{1}{r} \sum_{k} w_{k} e^{w_{k} t} \quad(r>1)$,

(10) $h(r, t)=\frac{\sin r \pi}{\pi} h^{*}(r, t) \quad$ where

(11) $h^{*}(r, t):=\int_{0}^{\infty} \frac{u^{r}}{\left|1+u^{r} e^{i r \pi}\right|^{2}} e^{-t u} d u$.

We have

(12) $\int_{0}^{\infty} h^{*}(r, t) d t=\int_{0}^{\infty} \frac{u^{r-1}}{\left|1+u^{r} e^{i r \pi}\right|^{2}} d u<\infty$

since the integrand on the right-hand side is $\sim u^{-r-1}$ for $u \rightarrow \infty$. Now observe that

(13) $\int_{0}^{\infty} h^{*}(r, t)\left(1-e^{-t}\right)^{n} d t=B_{n}^{*}(r)$,

(14) $\int_{0}^{\infty} h(r, t)\left(1-e^{-t}\right)^{n} d t=B_{n}(r)$. 
From (7) and (8) we infer

(15) $\frac{1}{z^{r}+1}=G(r, z)+H(r, z) \quad(\operatorname{Re} z>1)$

where

(16) $G(r, z):=\int_{0}^{\infty} g(r, t) e^{-z t} d t \quad\left(\operatorname{Re} z>\cos \frac{\pi}{r}, r>1\right)$,

(17) $H(r, z):=\int_{0}^{\infty} h(r, t) e^{-z t} d t \quad(\operatorname{Re} z \geq 0, r>0)$.

Let $r>1$. From (9) we deduce for $\operatorname{Re} z>\cos \frac{\pi}{r}$

$$
\begin{aligned}
G(r, z) & =-\frac{1}{r} \sum_{k} w_{k} \int_{0}^{\infty} \exp \left[\left(w_{k}-z\right) t\right] d t \\
G(r, z) & =-\frac{1}{r} \sum_{k} \frac{w_{k}}{z-w_{k}}
\end{aligned}
$$

From (12) we see that

(20) $H(r, z)$ is continuous for $\operatorname{Re} z \geq 0$ and holomorphic on $\operatorname{Re} z>0$.

From this we derive by analytic continuation and continuity the following facts:

(21) $\frac{1}{z^{r}+1}=H(r, z) \quad(\operatorname{Re} z \geq 0)$ for $0<r<1$,

(22) for $1<r<2$ the equation (15) with $G(r, z)$ given by (18) holds true for $\operatorname{Re} z \geq 0$ (note that $\cos \frac{\pi}{r}<0$ for $1<r<2$ ),

(23) for $r>2$ the equation (15) with $G(r, z)$ given by (18) holds true for $\operatorname{Re} z>\cos \frac{\pi}{r}$; in particular it holds true for $\operatorname{Re} z \geq 1$,

(24) for $r>1$ the equation (15) with $G(r, z)$ given by (19) holds true for real $z \geq 0$.

In particular,

(25) $1=\frac{1}{r} \sum_{k} 1+H(r, 0)$.

$0<r<1$ : From (21) and (14) we obtain

$$
S_{n}(r)=\sum_{m=0}^{n}\left(\begin{array}{l}
n \\
m
\end{array}\right)(-1)^{m} H(r, m)=B_{n}(r) .
$$

$\underline{1<r<2:}$ There are two zeros $w, \bar{w}$ where $w=w(r)$. From (22) we get

$$
\frac{1}{m^{r}+1}=-\frac{2}{r} \operatorname{Re} w \int_{0}^{\infty} \exp [(w-m) t] d t+H(r, m) \quad \text { for } m \in \mathbb{N}_{0}
$$


which implies

$$
S_{n}(r)=-\frac{2}{r} \operatorname{Re} w I(w,-1, n)+\sum_{m=0}^{n}\left(\begin{array}{l}
n \\
m
\end{array}\right)(-1)^{m} H(r, m) .
$$

By (5) and (14) this is equal to $A_{n}(r)+B_{n}(r)$.

$r>2$ : From (23) we get

$$
\frac{1}{m^{r}+1}=-\frac{1}{r} \sum_{k} w_{k} \int_{0}^{\infty} \exp \left[\left(w_{k}-m\right) t\right] d t+H(r, m) \quad \text { for } m \in \mathbb{N}
$$

From this together with (14) we infer

$$
S_{n}(r)=1-\frac{1}{r} \sum_{k} w_{k} \int_{0}^{\infty} e^{w_{k} t}\left[\left(1-e^{-t}\right)^{n}-1\right] d t+B_{n}(r)-H(r, 0) .
$$

We apply (6), (25) and (5) and obtain $S_{n}(r)=A_{n}(r)+B_{n}(r)$.

\section{Evaluation of $B_{n}^{*}(r)$, conclusion.}

Let $r>0, r \notin \mathbb{N}$. By definition we have

$$
B_{n}^{*}(r)=\int_{0}^{\infty} f_{r}(u) g_{n}(u) d u
$$

where

$$
f_{r}(u):=\frac{u^{r}}{\left|1+u^{r} e^{i r \pi}\right|^{2}}
$$

and

$$
g_{n}(u)=I(-u,-1, n)=\frac{n !}{\prod_{l=0}^{n}(u+l)}
$$

by $(5)$.

Put $u_{0}(r):=(1 / 2)^{1 / r}$ and split up

$$
B_{n}^{*}(r)=\left(\int_{0}^{u_{0}(r)}+\int_{u_{0}(r)}^{\infty}\right) \ldots d u=x+y .
$$

Estimation of $y$. Write $g_{n}(u)=u^{-1} h_{n}(u)$. Then

$$
y \leq h_{n}\left(u_{0}(r)\right) \int_{0}^{\infty} f_{r}(u) u^{-1} d u .
$$


Since

$$
h_{n}\left(u_{0}(r)\right)=u_{0}(r) n^{-u_{0}(r)}\left(n^{u_{0}(r)} g_{n}\left(u_{0}(r)\right)\right)
$$

and $n^{u_{0}(r)} g_{n}\left(u_{0}(r)\right) \rightarrow \Gamma\left(u_{0}(r)\right)$ we get $y \ll n^{u_{0}(r)}$.

Estimation of $x$. For $0 \leq u \leq u_{0}(r)$ one has

$$
\begin{aligned}
\frac{1}{\left|1+u^{r} e^{i r \pi}\right|^{2}} & =1+O\left(u^{r}\right), \\
g_{n}(u) & =n^{-u} \Gamma(u)\left(1+O\left(\frac{1}{n}\right)\right), \\
\Gamma(u) & =\frac{1}{u}(1+O(u))
\end{aligned}
$$

with $O$-constants depending on $r$ at most.

We conclude that

$$
f_{r}(u) g_{n}(u)=u^{r-1} \exp (-u \log n)\left(1+O\left(u^{r}+u+\frac{1}{n}\right)\right)
$$

for $0 \leq u \leq u_{0}(r)$. From this it follows that

$$
x=\Gamma(r)(\log n)^{-r}+O\left((\log n)^{-2 r}+(\log n)^{-r-1}\right) .
$$

It remains to show that $T_{n}(r):=-S_{n}(r) \downarrow 0\left(n \rightarrow \infty, n \geq n_{0}(r)\right)$ in the case $1<r<2$. We have

$$
T_{n}(r)-T_{n+1}(r)=\left(A_{n+1}(r)-A_{n}(r)\right)+\left(-\frac{\sin r \pi}{\pi}\right)\left(B_{n}^{*}(r)-B_{n+1}^{*}(r)\right) .
$$

Since

$$
A_{n}(r)=\frac{2}{r} \operatorname{Re}\left[\Gamma(1-w(r)) n^{w(r)}\right]+O\left(n^{\operatorname{Re} w(r)-1}\right)
$$

we get $A_{n+1}(r)-A_{n}(r) \ll n^{\cos \frac{\pi}{r}-1}$. From the representation

$$
B_{n}^{*}(r)-B_{n+1}^{*}(r)=\int_{0}^{\infty} \frac{u^{r}}{\left|1+u^{r} e^{i \pi r}\right|^{2}} I(-(u+1),-1, n) d u
$$

and

$$
\begin{aligned}
I(-(u+1),-1, n) & =\frac{1}{u+1} \prod_{l=1}^{n}\left(1+\frac{u+1}{l}\right)^{-1} \\
& \geq \frac{1}{u+1} \exp [-(u+1) L(n)], \quad L(n):=\log n+1 \\
& \geq \frac{1}{e n} \frac{1}{u+1} \exp [-u L(n)]
\end{aligned}
$$


we obtain

$$
\begin{aligned}
B_{n}^{*}(r)-B_{n+1}^{*}(r) & \geq \frac{1}{8 e n} \int_{0}^{1} u^{r} \exp (-u L(n)) d u \\
& =\frac{1}{8 e n}\left(\frac{\Gamma(r+1)}{L(n)^{r+1}}-\int_{0}^{\infty} v^{r} e^{-v} d v\right) \\
& \geq \frac{1}{8 e n}\left(\frac{\Gamma(r+1)}{L(n)^{r+1}}-\frac{L(n)^{r}}{n}\right) .
\end{aligned}
$$

We conclude that

$$
T_{n}(r)-T_{n+1}(r) \geq\left(-\frac{\sin r \pi}{\pi}\right) \frac{\Gamma(r+1)}{16 e} \frac{1}{n L(n)^{r+1}}+O\left(n^{\cos \frac{\pi}{r}-1}\right) .
$$

Since $\cos \frac{\pi}{r}<0$ the expression on the right-hand side is positive for $n \geq n_{0}(r)$.

5. Final remarks. We can see from the proofs above that the queer behaviour of $S_{n}(r)$ is determined mainly by the zeros of the function $z^{r}+1$ in $\mathbb{C}^{-}$. These zeros which appear in the term $A_{n}(r)$ are the reason for the quite complicated structure of $S_{n}(r)$.

If we replace the term $m^{r}+1$ in the definition of $S_{n}(r)$ by $m^{r}$ and consider

$$
\widetilde{S}_{n}(r):=\sum_{m=1}^{n}\left(\begin{array}{c}
n \\
m
\end{array}\right) \frac{(-1)^{n}}{m^{r}}
$$

the situation is much simpler. Indeed, we can write

$$
\begin{aligned}
\widetilde{S}_{n}(r) & =\sum_{m=1}^{n}\left(\begin{array}{c}
n \\
m
\end{array}\right)(-1)^{m} \frac{1}{\Gamma(r)} \int_{0}^{\infty} t^{r-1} e^{-m t} d t \\
& =-\frac{1}{\Gamma(r)} \int_{0}^{\infty} t^{r-1}\left[1-\left(1-e^{-t}\right)^{n}\right] d t \\
& =-\frac{1}{\Gamma(r)} \sum_{m=0}^{n-1} J_{m}(r)
\end{aligned}
$$

with $J_{m}(r):=\int_{0}^{\infty} t^{r-1}\left(1-e^{-t}\right)^{m} e^{-t} d t$. It is not difficult to see that

$$
J_{m}(r)=\frac{[\log (m+1)]^{r-1}}{m+1}\left[1+O\left(\frac{1}{\sqrt{\log (m+1)}}\right)\right]
$$

and consequently

$$
\lim _{n \rightarrow \infty} \frac{\widetilde{S}_{n}(r)}{(\log n)^{r}}=-\frac{1}{\Gamma(r)} .
$$




\section{References}

[1] I. S. Gradshteyn, I. M. Ryzhik, Table of integrals, series and products. Academic Press, New York etc. 1980.

[2] A. Hiller, Kostengünstigste zuverlässige Inspektionsstrategien zur Abwehr kombinierter illegaler Aktivitäten. Dissertation. NWF I - Mathematik. Universität Regenburg 1990.

[3] O. Perron, Irrationalzahlen. 4. Auflage, de Gruyter, Berlin 1960.

[4] E. C. Titchmarsh, Introduction to the theory of Fourier integrals. Second edition. Oxford University Press, Oxford 1948.

Robert Denk, Richard Warlimont

NWF I - Mathematik

Universität Regensburg

D-93040 Regensburg

Germany 